\section{Ubiquitin-Proteasome System}

\section{Synonyms}

UPS

\section{Definition}

A specialized system in cells for regulating their protein composition. Degraded, misfolded, or aberrant proteins are tagged by ubiquitylation (attachment of ubiquitin to a substrate protein) for removal by digestion at the cytoplasmic 20/26S proteasome enzyme complex.

\section{Ultrasonic}

\section{Definition}

Refers to sound energies having a frequency above the human hearing range. The highest frequency that the human ear can detect is approximately 20,000 cycles per second $(20,000 \mathrm{~Hz})$.

Attachment bonds signaled by ultrasonic vocalizations have been proposed to underlie a variety of social relationships, for example, parent-infant, filial, and pair (male-female) bond formation. These are all typically characterized by preferential proximity seeking and all involve a response to separation. These forms of attachment appear to be common to many species including humans, suggesting that the neural basis can be investigated in animal models. Emerging evidence suggests that the biology of attachment in its many forms may also be similar across species.

\section{Ultrasonic Vocalizations}

Paul B. S. Clarke and Jennifer Wright

Department of Pharmacology and Therapeutics, McGill University, Montréal, QC, Canada

\section{Synonyms}

Ultrasonic call; Ultrasonic vocalization

\section{Definition}

Ultrasonic vocalizations (USVs) occur at sound frequencies above the normal limit of human hearing, i.e., $20 \mathrm{kHz}$. Rodent ultrasonic vocalizations are generated by the larynx and are abolished by surgical denervation of this organ. 